\title{
Catalytic Living Ring Opening Metathesis Polymerisation: The Importance of Ring Strain in Chain Transfer Agents
}

\author{
Peng Liu ${ }^{+}$, Mohammad Yasir ${ }^{+}$, and Andreas F. M. Kilbinger*
}

\begin{abstract}
A recently developed catalytic living ring opening metathesis polymerisation (ROMP) was investigated using a series of reversible chain transfer agents (CTA) carrying either cyclopentene or cyclohexene rings, differing only in ring strain. All cyclopentene derivatives examined showed significantly faster reaction rates than the corresponding cyclohexene derivatives. This resulted in lower molecular weight dispersities and better control of the molecular weight for the cyclopentene compared to the cyclohexene CTAs. Both Grubbs' second and third generation catalysts could be employed in catalytic living ROMP using cyclopentene CTA derivatives. The kinetics of different CTAs were studied, block copolymers were synthesised and residual ruthenium quantified by ICP-OES. All polymers were fully characterised by NMR, GPC and MALDI-ToF mass spectrometry. The new cyclopentene CTAs are readily synthesised in a few straightforward steps and provide faster reaction kinetics than all previously reported reversible CTAs.
\end{abstract}

Ring Ring opening metathesis polymerisation (ROMP), which uses the metathesis of cyclic olefins to synthesise linear polymers, has become a very important method for polymer synthesis in biomedical, material, academic and industrial applications. ${ }^{[1-8]}$ Living ROMP, wherein irreversible chaintransfer events and termination reactions are absent, is an important polymerisation technique, due to its excellent control of molecular weight and the narrow molecular weight distribution of the resulting polymers. ${ }^{[9,10]}$ For polymerisations, ruthenium carbene complexes developed by Grubbs et al. (G2: (1,3-bis(2,4,6-trimethylphenyl)-2-imidazolidinylidene) dichloro (phenylmethylene)(tricyclohexylphosphine)ruthenium and G3: dichloro[1,3-bis(2,4,6-trimethylphenyl)-2imidazolidinylidene] (benzylidene)bis(3-bromopyridine)ruthenium(II)) are frequently used mainly due to their easy handling and high functional group tolerance. However, in a typical living ROMP polymerisation, the ruthenium catalyst will always be covalently bound to the end of the growing polymer chain. It is, therefore, mechanistically determined that each catalyst can only form one polymer chain, which means stoichiometric amounts of the transition metal complex are required with respect to the number of polymer

[*] P. Liu, ${ }^{[+]}$M. Yasir, ${ }^{[+]}$A. F. M. Kilbinger Department of Chemistry, University of Fribourg Chemin du Musée 9, 1700 Fribourg (Switzerland) E-mail: andreas.kilbinger@unifr.ch

$\left.{ }^{+}\right]$These authors contributed equally to this work. chains formed in a living ROMP. This can result in high catalyst loadings, especially during the synthesis of low molecular weight polymers, which is expensive and leads to potentially toxic ruthenium residues in these polymers.

Numerous methods have been developed to reduce the level of catalyst in the final metathesis products. ${ }^{[11-26]}$ However, these methods mostly rely on easy removal of the transition metal complex or improved product separation. To date, only few reports exist on catalytic metathesis polymerisations producing multiple polymer chains per ruthenium complex. Acyclic diene metathesis polymerisation (ADMET), pioneered by Wagener et al. uses catalytic amounts of metal complex ${ }^{[27-29]}$ in a step-growth process. Homotelechelic polymers prepared by ruthenium complexes were first described by Grubbs and co-authors using an irreversible chain transfer agent (CTA) in the presence of sub-stoichiometric quantities of ruthenium complex. ${ }^{[30-35]}$ Fewer examples have been reported on the synthesis of heterotelechelic polymers using only catalytic quantities of transition metal complex. ${ }^{[36-39]}$ However, none of the examples given above can be considered as living, as molecular weight distributions are typically broad, and block copolymers cannot be made.

We recently described a mechanism for catalytic living ROMP that relies on a degenerative reversible chain-transfer polymerisation that required only a catalytic amount of ruthenium carbene complex. ${ }^{[40]}$ Here, we report new CTAs (Figure 1) based on either cyclopentene or cyclohexene derivatives and provide a kinetic analysis that shows the importance of ring strain in CTAs for degenerative chain transfer metathesis polymerizations.

Catalytic living $\mathrm{ROMP}^{[40]}$ is based on a degenerative chain transfer mechanism in which a sub-stoichiometric quantity of
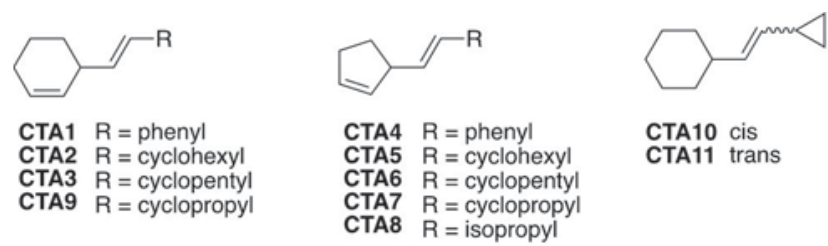

CTA10 cis CTA11 trans CTA9 $R=$ cyclopropyl
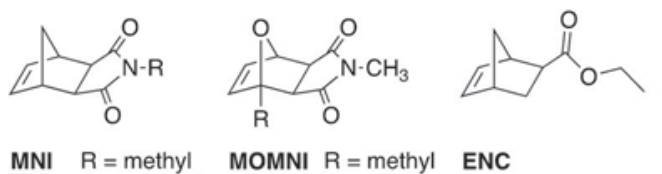

MOMNI R $=$ methyl ENC
EOMNI $R=$ ethyl

$\begin{array}{ll}\text { MNI } & R=\text { methyl } \\ \text { HNI } & R=n \text {-hexyl }\end{array}$

$\begin{array}{ll}\text { HNI } & R=n \text {-hexyl } \\ \text { PNI } & R=\text { phenyl }\end{array}$

Figure 1. Top: Chain transfer agents (CTA1-CTA9) and model compounds CTA10-11. Bottom: Monomers (MNI, HNI, PNI, MOMNI, EOMNI, ENC, NBSM) investigated in this study. 
A

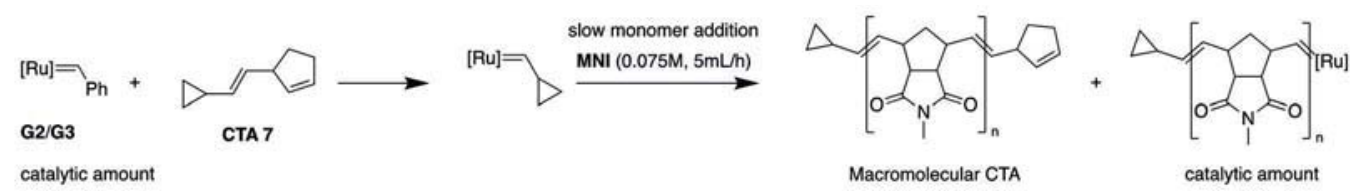

B
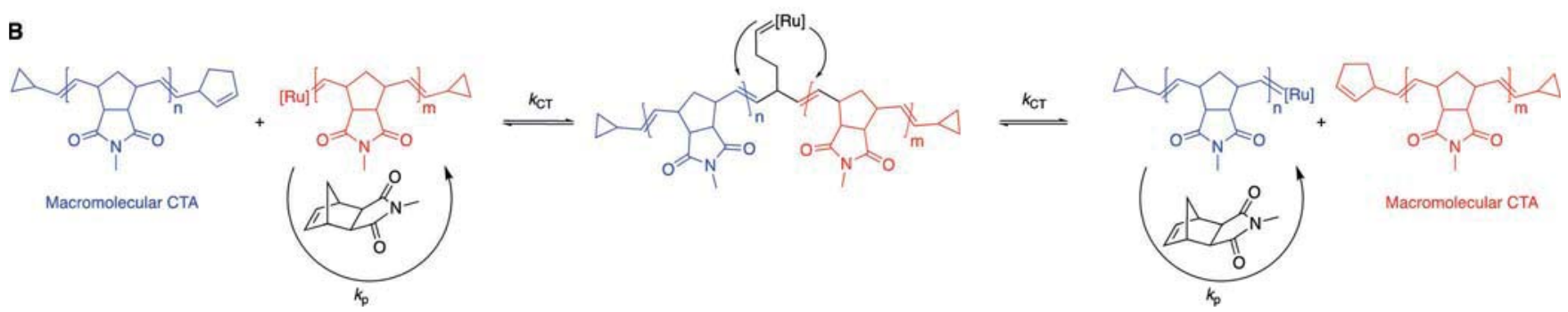

Figure 2. A) Polymerization of MNI in the presence of CTA7 under slow monomer addition conditions yields mainly polymer chains being endfunctionalized with the cyclopentenyl unit and a small number (catalytic amount) of propagating chains. B) The degenerative chain transfer mechanism ${ }^{[4]}$ exploited in this investigation shown as an example using CTA7 and MNI. The polymer end group of the macromolecular CTA (shown in blue) exchanges with the propagating carbene end group (polymer shown in red) via an intermediate (right) in a degenerate equilibrium. $k_{\mathrm{p}}$ : propagation rate constant, $k_{\mathrm{CT}}$ : chain transfer rate constant. $[\mathrm{Ru}]=\mathbf{G} \mathbf{2}$ or $\mathbf{G} 3$ based propagating carbene complexes.

a propagating ruthenium carbene chain end of a polymer exchanges rapidly with other chain ends. This results in quasisimultaneous growth of all polymer chains leading to narrow molecular weight distributions and control of molecular weight (see Figure 2B as an example with CTA7 and MNI).

As previously reported, CTA1 ${ }^{[40]}$ (Figure 1) reacts only very slowly with propagating ruthenium alkylidene complexes. It is for this reason that it cannot be employed as a chain transfer agent in catalytic living ROMP. In order to assign the low reactivity of CTA1 to the cyclohexenyl group, CTA2-3 were synthesized both of which carried the same endocyclic double bond as CTA1 but different substituents (cyclohexyl and cyclopentyl groups) attached to the exocyclic double bond. ${ }^{[41]}$ However, neither CTA2 nor CTA3 show a higher reactivity than CTA1 in time-resolved ${ }^{1} \mathrm{H}-\mathrm{NMR}$ spectroscopic experiments (see Supporting Information). As the low reactivity of CTA1-3 is caused by the endocyclic double bond, common to all three CTAs, we decided to synthesize a series of new CTAs carrying endocyclic double bonds within five-membered rings (CTA4-8) in which the ring strain was assumed to be higher. In addition to the common cyclopentenyl group, CTA4-8 differ in the substituent attached to the exocyclic double bond. CTA4 carries a phenyl group attached to the exocyclic double bond and is therefore similar in structure to CTA1, whereas CTA5 represents the saturated version of CTA4, carrying a cyclohexyl substituent attached to the exocyclic double bond.

In a catalytic living ROMP a commercial ruthenium benzylidene complex first reacts with a reversible chain transfer agent (for example CTA4-8) yielding a new ruthenium carbene complex (carrying the group R, Figure 1 CTA4-8). A strained cyclic monomer is then added slowly to this mixture of CTA and newly formed ruthenium carbene complex (Figure 2A). Due to the low monomer concentration, the propagation reaction and reaction with the CTA become equally likely, leading to a reversible chain transfer equilibrium (Figure 2B) in which the polymer end groups of two chains exchange as described previously. ${ }^{[40]}$
To investigate the reactivity of CTA4-5 we followed the reaction of propagating MNI and CTA4-5 by ${ }^{1} \mathrm{H}-\mathrm{NMR}$ spectroscopy (addition of 10 equiv of CTA4 or CTA5 to a G3 initiated MNI (34 equiv) solution). We observed a fast and complete conversion of the signal corresponding to the propagating MNI alkylidene $(18.50 \mathrm{ppm})$ to the new carbene (a benzylidene (19.06 ppm) for CTA4 and cyclohexylmethylidene (18.97 ppm) for CTA5). We furthermore observed that over time the G3-benzylidene (from CTA4) decomposed less rapidly than the G3-cyclohexylmethylidene (from CTA5). This initial investigation showed clearly that CTA4-5 carrying the more strained cyclopentenyl groups were dramatically more reactive than the cyclohexenyl carrying CTA1-3. Despite the greater tendency to open the five-membered ring, no ruthenium-alkylidene signal could so far be recorded that would correspond to the ring-opened intermediate indicating that such alkylidenes represent transient structures that immediately undergo ring closing reactions.

Next, three further CTAs (CTA6-8) were investigated, all carrying the ring strained cyclopentenyl group but yielding different ruthenium alkylidenes upon reaction with the propagating G3 complex (addition of 10 equiv of either CTA6,7 or 8 to a G3 initiated MNI (34 equiv) solution). It could be shown that CTA6-8 all gave rapid and complete conversions to the new alkylidene signals when followed by ${ }^{1} \mathrm{H}-\mathrm{NMR}$ spectroscopy. The stability of the newly formed ruthenium alkylidenes also varied significantly and decreased in the following order: Ru-cyclopropylmethylidene (17.20 ppm, from CTA7) > Ru-benzylidene (19.04 ppm, from CTA4) > Ru-cyclopentylmethylidene (18.58 ppm, from CTA6) > Ru-cyclohexylmethylidene (18.97 ppm, from CTA5) $=$ Ru-isopropylidene (18.66 ppm, from CTA8). However, in any catalytic living ROMP reaction these stability differences are not important as these ruthenium carbene complexes only exist for a very short time at the preequilibrium stage (Figure $2 \mathrm{~A}$ ).

In our previous investigation ${ }^{[40]}$ we could show that our CTA reacted exclusively via the "endocyclic double bond 
first" pathway. CTA7 carries a cyclopropyl group attached to the exocyclic double bond that is sterically less demanding than the residues of all other CTAs investigated here or previously. To investigate whether CTA7 reacts via the edocyclic or exocyclic double bond first, we synthesized model compounds CTA10 (cis) and CTA11 (trans) differing in their exocyclic double bond configuration but lacking the endocyclic double bond altogether.

Time-resolved ${ }^{1} \mathrm{H}-\mathrm{NMR}$ spectroscopic experiments revealed (Supporting Information), however, that when a propagating (MNI) G3 catalyst (1 equiv) was exposed to CTA9-11 (10 equiv) CTA10 formed the new cyclopropylmethylidene carbene with $90 \%$ conversion after 24 min. whereas CTA11 only formed $64 \%$ (24 min.) and CTA9 $62 \%$ (24 min.) of the new carbene. The cyclohexene carrying CTA9 therefore shows reaction rates on the same order of magnitude as the cross metathesis with the trans configured CTA11. CTA7, however, forms the new cyclopropylmethylidene under identical reaction conditions with a conversion of $100 \%$ in $8 \mathrm{~min}$. As the steric demand of cyclopentyl or cyclohexyl groups is very similar, ${ }^{[42]}$ we estimated that the steric hindrance of the exocyclic double bond in CTAs7,9 and $\mathbf{1 1}$ was also similar. This would suggest that a very different mechanism, most likely the ring opening-ring closing sequence is responsible for this massively increased reaction rate.

Encouraged by these results, we next carried out the polymerisation of MNI under catalytic living ROMP conditions (see Supporting Information). Since the rate of propagation of ring-strained monomers, such as norbornenes, is significantly faster than the olefin metathesis reaction with the CTA $\left(k_{\mathrm{CT}} \ll k_{\mathrm{p}}\right.$, see Figure $\left.2 \mathrm{~B}\right),{ }^{[43]}$ the monomer concentration was kept low at all times, which was realised by slow addition via a syringe pump. Reaction conditions were optimised using CTA5.

When MNI $\left(0.075 \mathrm{molL}^{-1}\right)$ was added slowly $\left(5 \mathrm{~mL} \mathrm{~h}^{-1}\right)$ to the solution of Grubbs' third-generation catalyst $(\mathbf{G 3})$ and CTA5, the desired polymer was formed (polymer 1, $M_{\mathrm{nGPC}}$ $\left(\mathrm{CHCl}_{3}\right)=7100 \mathrm{~g} \mathrm{~mol}^{-1}, \quad Ð=1.18, \quad \mathrm{M}_{\text {peak }} \quad(\mathrm{MALDI}-\mathrm{ToF})=$ $\left.4001.71 \mathrm{~g} \mathrm{~mol}^{-1}\right)$. An isotopically resolved MALDI-ToF mass spectrum matching the polymer structure with both CTA5 end-groups (cyclohexyl and cyclopentenyl) is shown in Figure 3.

As expected, the molecular weight of the produced polymer was much lower than that calculated from the monomer:G3 ratio $\left(M_{\mathrm{n}}=53100 \mathrm{~g} \mathrm{~mol}^{-1}\right)$ indicating that successful reversible chain transfer had taken place. In case of a non-catalytic polymerisation, the molecular weight of the polymer would have been significantly higher (see Table 1). All of the optimisation results can be found in the Supporting Information. Similar results were obtained when Grubbs' second-generation catalysts (G2) was used under the optimised reaction conditions (Supporting Information).

In order to compare the efficiency of CTA1-8 we carried out eight different catalytic living ROMP polymerizations using monomer MNI (see Table 1 and Supporting Information). As expected, CTA1-3 (entries 1-3, Table 1) did not yield polymers (polymer 3-5) with controlled molecular weights (see above). Instead, the molecular weights obtained

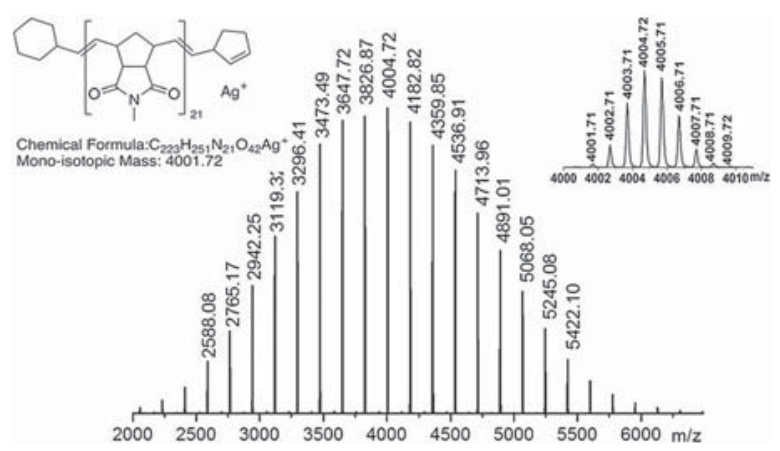

Figure 3. MALDI-ToF mass spectrum (DCTB, AgTFA) of polymer 1. Left: Chemical structure of polymer 1 with calculated mono-isotopic mass. Center: Mass distribution. Right: Most intense peak of the distribution, isotopically resolved.

Table 1: Catalytic Living ROMP using CTA1-8. ${ }^{[a]}$

\begin{tabular}{llllllll}
\hline$\#$ & Polymer & CTA & $M_{\text {ncalc. }}{ }^{[b]}$ & $M_{\text {ncalc. }}{ }^{[c]}$ & $M_{\text {nobs. }}{ }^{[\mathrm{d}]}$ & $\bigoplus^{[\mathrm{d}]}$ & Yield [\%] \\
\hline 1 & polymer 3 & CTA1 & 53100 & 5310 & 41000 & 1.29 & 99 \\
2 & polymer 4 & CTA2 & 53100 & 5310 & 38000 & 1.35 & 99 \\
3 & polymer 5 & CTA3 & 53100 & 5310 & 34000 & 1.25 & 99 \\
4 & polymer 6 & CTA4 & 53100 & 5310 & 9900 & 1.18 & 99 \\
5 & polymer 1 & CTA5 & 53100 & 5310 & 7100 & 1.18 & 98 \\
6 & polymer 7 & CTA6 & 53100 & 5310 & 7800 & 1.16 & 99 \\
7 & polymer 8 & CTA7 & 53100 & 5310 & 8900 & 1.17 & 99 \\
8 & polymer 9 & CTA8 & 53100 & 5310 & 9000 & 1.17 & 99 \\
\hline
\end{tabular}

[a] All of the reactions were carried out with $4.45 \mathrm{mg} \mathrm{G} 3(0.005 \mathrm{mmol})$ and 10 equiv of CTA in $1.5 \mathrm{~mL}$ degassed DCM, followed by 300 equiv of MNI (266 mg. $1.5 \mathrm{mmol}$ ), which was dissolved in $20 \mathrm{~mL}$ degassed DCM $\left(0.075 \mathrm{~mol} \mathrm{~L}^{-1}\right)$ added by a syringe pump at $5 \mathrm{mLh}^{-1}$ under Ar. [b] $M_{n}$ under non-catalytic conditions was calculated with a degree of polymerization $X_{n}=$ [monomer]:[G3] $=300: 1$. [c] $M_{n}$ under catalytic living ROMP conditions was calculated using a degree of polymerization $X_{n}=[$ monomer]: $[C T A]=30: 1$. [d] Determined by GPC (chloroform). Note that entries 1-3 employing cyclohexenyl carrying CTA1-3 did not result in any molecular weight control.

for these CTAs resembled those of classical living ROMP emphasizing once more that the chain transfer to CTA1-3 is too slow to control the molecular weight in a catalytic fashion. CTA4-8, on the other hand, gave excellent molecular weight control of the resulting polymers (polymer1, polymer 6-9) and narrow molecular weight dispersities. Isotopically resolved MALDI-ToF mass spectra matched the masses for polymers with end groups corresponding to the CTA employed (see Supporting Information).

To expand the applicability of catalytic living ROMP, different monomers such as exo- $N$-hexyl norbornene imide (HNI, Figure 1), exo- $N$-phenyl norbornene imide (PNI, Figure 1), endo-5-norbornene-2,3-bis(triisopropyl)silylmethanol (NBSM, Figure 1) and exo-ethyl-5-norbornene carboxylate (ENC, Figure 1) were polymerised under catalytic living ROMP conditions using CTA5 or CTA7. The isotopically resolved MALDI-ToF MS spectra of all polymers matched the two expected end groups of CTA5 or CTA7 (see Supporting Information, polymer 10-13).

It is furthermore worth noting that when we used exo- $N$ methyl-7-oxabicyclo[2.2.1] hept-4-methyl-5-ene-2,3-dicarboximide (MOMNI, Figure 1) and exo- $N$-methyl-7-oxabicyclo- 
[2.2.1]hept-4-ethyl-5-ene-2,3-dicarboximide

(EOMNI, Figure 1), carrying sterically demanding groups on the bridge head carbon that slow down propagation, polymerisations could be run in one pot (see Supporting Information, polymer 14-15) without the need of a slow monomer addition via a syringe pump. This feature will be further investigated in the near future.

To prove that the monomer:CTA ratio determines the polymer molecular weight and that the polymerisation fulfils living characteristics, different molecular weight polymers were synthesised by changing the MNI:CTA5 ratio (Figure 4). A linear correlation between the molecular weight (Mn, GPC) and the MNI:CTA5 ratio was obtained as expected for a living polymerization under reversible chain transfer conditions.

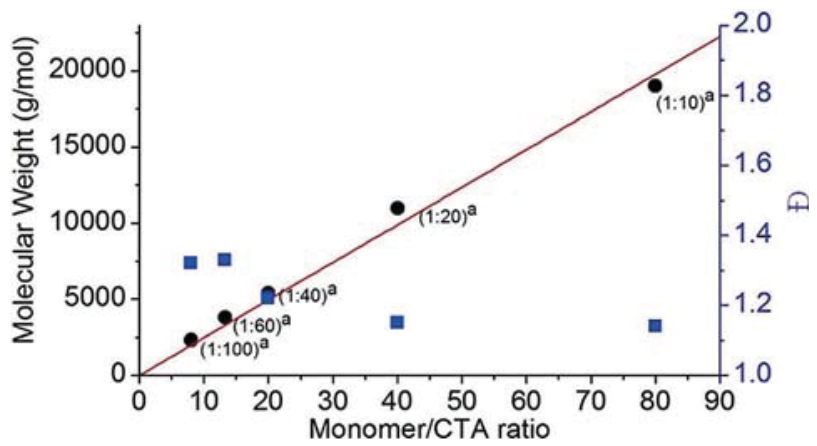

Figure 4. Plot of the molecular weight ( $\mathrm{Mn}, \mathrm{GPC}\left(\mathrm{CHCl}_{3}\right)$, black circles) and molecular weight dispersity $(\Theta$, blue squares) versus the [MNI]/ [CTA5] ratio. [a] The ratios reported in brackets denote the [G3]/[CTA5] ratio.

The molecular weight dispersities of the resulting polymers was low $(Ð=1.2-1.3)$ and the masses of the polymers matched the assumed structures with CTA5 end groups (see above) which provides a strong indication that the polymerisation process is indeed living (Figure 4, see also Supporting Information polymer 16-20).

Furthermore, the G3:CTA5 ratio was also varied during the experiment described above (see ratios in brackets in Figure 4). For low molecular weight polymers a ratio of CTA5:G3 = 100 was used, in other words, one molecule of $3^{\text {rd }}$ generation Grubbs catalyst was responsible for the synthesis of 100 living polymer chains. This once more emphasises the significant reduction of the amount of transition metal possible with this catalytic method.

One important feature of a living polymerisation is the ability to prepare block copolymers. To further prove that our newly developed chain transfer agents produced living polymers, monomers MNI (300 equiv, $0.075 \mathrm{~mol} \mathrm{~L}^{-1}$ ) and HNI (300 equiv, $\left.0.075 \mathrm{~mol} \mathrm{~L}^{-1}\right)$ were added $\left(5 \mathrm{mLh}^{-1}\right)$ to the G3/CTA5 (1:10) mixture sequentially. GPC (chloroform) analysis performed after addition of both monomers showed a monomodal molecular weight distribution and a molecular weight of $17900 \mathrm{~g} \mathrm{~mol}^{-1}$ (polymer 21, Supporting Information) supporting a block copolymer formation. A more rigorous proof for the proposed degenerate chain transfer mechanism and block copolymer formation was obtained when the polymer taken from the catalytic living ROMP of MNI, CTA5 and G3 was precipitated, dried and analysed and subsequently redissolved and exposed to HNI (300 equiv, $\left.0.075 \mathrm{molL}^{-1}, 5 \mathrm{mLh}^{-1}\right)$ and $\mathbf{G 3}(0.005 \mathrm{mmol})$ for the growth of a second polymer block (Figure 5). GPC characterisation after polymerizing the first $\left(M_{\mathrm{nGPC}}\left(\mathrm{CHCl}_{3}\right)=7100 \mathrm{~g} \mathrm{~mol}^{-1}\right.$, $Ð=1.18)$ and second monomer $\left(M_{\mathrm{nGPC}} \quad\left(\mathrm{CHCl}_{3}\right)=\right.$ $22500 \mathrm{~g} \mathrm{~mol}^{-1}, Ð=1.36$ ) revealed the formation of a diblock copolymer (Figure 5).

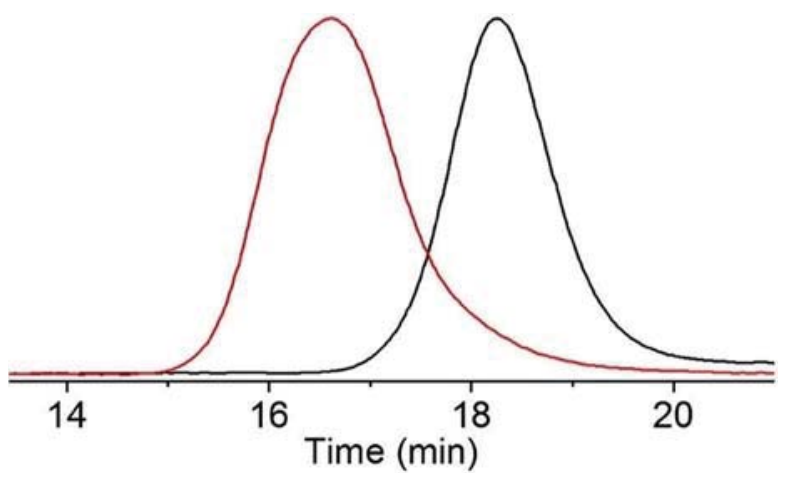

Figure 5. GPC trace of polymer 1 (black, $M_{\mathrm{nCPC}}\left(\mathrm{CHCl}_{3}\right)=7100 \mathrm{~g} \mathrm{~mol}^{-1}$, $\bigoplus=1.18$ ) and block copolymer polymer 22 (red, $M_{\mathrm{nCPC}}$ $\left.\left(\mathrm{CHCl}_{3}\right)=22500 \mathrm{~g} \mathrm{~mol}^{-1}, Ð=1.36\right)$. Catalyst $\mathbf{G} 3$ and CTA5 were employed for this reaction.

Polymers obtained by catalytic living ROMP are noticeably less coloured than those obtained by classical living ROMP (Supporting Information). To quantify the residual ruthenium in our catalytic living ROMP polymer, two similar molecular weight and dispersity polymers were synthesised by catalytic and classical living ROMP (Supporting Information). To emphasise the efficiency of our method: only $33 \mathrm{mg}$ of polymer $\left(M_{\mathrm{nGPC}}\left(\mathrm{CHCl}_{3}\right)=6900 \mathrm{~g} \mathrm{~mol}^{-1}, Ð=1.15\right)$ could be synthesised non-catalytically using $4.45 \mathrm{mg}$ of $\mathbf{G 3}$, while $266 \mathrm{mg}$ of polymer $\left(M_{\mathrm{nGPC}}\left(\mathrm{CHCl}_{3}\right)=7200 \mathrm{~g} \mathrm{~mol}^{-1}, Ð=1.20\right)$ was synthesised via catalytic living ROMP using the same amount of G3. Furthermore, inductively coupled plasma optical emission spectroscopy (ICP-OES) showed that $1.38 \mathrm{ppm}$ of ruthenium remained in $3.5 \mathrm{mg}$ of the polymer prepared non-catalytically after two precipitations in cold methanol whereas only $0.21 \mathrm{ppm}$ ruthenium was left in $3.5 \mathrm{mg}$ of the catalytically prepared polymer sample (Supporting Information). This result sums up several advantages of the catalytic living ROMP method, as with the same amount of G3 complex, more polymer at lower cost and with lower residual transition metal contamination can be prepared.

In conclusion, we have developed a series of new chain transfer agents which can be prepared in good yields and few straightforward steps for a recently developed catalytic living ring opening metathesis polymerisation method. Kinetic investigations of the chain transfer agents clearly showed that their efficiency during the degenerate chain transfer process depends highly on their ring strain. While the cyclopentenyl carrying chain transfer agents yielded wellcontrolled polymers with low molecular weight dispersities, cyclohexenyl carrying chain transfer agents exhibited only 
low reactivity. Grubbs' second and third generation catalysts were examined in catalytic living ROMP and produce polymers with the expected end groups which are mechanistically defined by the chain transfer agent employed. Block copolymers could be synthesised either via continuous slow addition of two different monomers or via chain extension of a separately prepared macromolecular chain transfer agent. The lowest ruthenium catalyst-to-CTA ratio examined in this report was 1:100. This equates to a 100-fold saving of ruthenium carbene complex compared to a classical living ROMP. The residual ruthenium in the final polymer was also analysed by ICP-EOS and found to be significantly reduced compared to non-catalytically prepared metathesis polymers. Catalytic living ROMP combined with the simplified chain transfer agent synthesis reported here offers a huge potential especially for the synthesis of functional polymers for biomedical, materials, industrial and academic use where very low ruthenium contamination is crucial.

\section{Acknowledgements}

The authors thank the Swiss National Science Foundation for financial support.

\section{Conflict of interest}

The authors declare no conflict of interest.

Keywords: chain transfer agents .

living catalytic polymerization - olefin metathesis .

ring opening polymerization $\cdot$ ring strain

[1] P. Liu, C. Ai, Ind. Eng. Chem. Res. 2018, 57, 3807-3820.

[2] Y. Chen, M. M. Abdellatif, K. Nomura, Tetrahedron 2018, 74, $619-643$.

[3] C. Slugovc in Industrial applications of olefin metathesis polymerization in Olefin Metathesis (Ed.: K. Grela), Wiley, Hoboken, 2014, Chap. 10, pp. 329-333.

[4] A. E. Madkour, A. H. R. Koch, K. Lienkamp, G. N. Tew, Macromolecules 2010, 43, 4557-4561.

[5] K. D. Camm, D. E. Fogg, NATO Sci. Ser. II 2007, 243, 285-303.

[6] S. Hilf, A. F. M. Kilbinger, Nat. Chem. 2009, 1, 537-546.

[7] J. M. Fishman, L. L. Kiessling, Angew. Chem. Int. Ed. 2013, 52, 5061-5064; Angew. Chem. 2013, 125, 5165-5168.

[8] E. M. Kolonko, J. K. Pontrello, S. L. Mangold, L. L. Kiessling, J. Am. Chem. Soc. 2009, 131, 7327-7333.

[9] K. Nomura, M. M. Abdellatif, Polymer 2010, 51, 1861-1881.

[10] S. Sutthasupa, M. Shiotsuki, F. Sanda, Polym. J. 2010, 42, $905-$ 915.

[11] H. Clavier, K. Grela, A. Kirschning, M. Mauduit, S. P. Nolan, Angew. Chem. Int. Ed. 2007, 46, 6786-6801; Angew. Chem. 2007, 119, 6906-6922.

[12] W. J. Sommer, M. Weck, Coord. Chem. Rev. 2007, 251, 860-873.

[13] M. R. Buchmeiser, Chem. Rev. 2009, 109, 303-321.

[14] J. H. Cho, B. M. Kim, Org. Lett. 2003, 5, 531-533.

[15] H. Wang, S. N. Goodman, Q. Dai, G. W. Stockdale, W. M. Clark, Org. Process Res. Dev. 2008, 12, 226-234.
[16] H. D. Maynard, R. H. Grubbs, Tetrahedron Lett. 1999, 40, 4137 4140.

[17] Y. M. Ahn, K. L. Yang, G. I. Georg, Org. Lett. 2001, 3, 14111413.

[18] L. A. Paquette, J. D. Schloss, I. Efremov, F. Fabris, F. Gallou, J. M. Andino, J. Yang, Org. Lett. 2000, 2, 1259-1261.

[19] J. Méndez-Andino, L. A. Paquette, Org. Lett. 2000, 2, $1263-$ 1265.

[20] D. W. Knight, I. R. Morgan, A. J. Proctor, Tetrahedron Lett. 2010, 51, 638-640.

[21] W. Liu, P. J. Nichols, N. Smith, Tetrahedron Lett. 2009, 50, $6103-$ 6105.

[22] A. Löber, A. Verch, B. Schlemmer, S. Höfer, B. Frerich, M. R. Buchmeiser, Angew. Chem. Int. Ed. 2008, 47, 9138-9141; Angew. Chem. 2008, 120, 9278-9281.

[23] N. K. Yee, V. Farina, I. Houpls, N. Haddad, R. P. Frutos, F. Gallou, X. J. Wang, X. Wei, R. D. Simpson, X. Feng, V. Fuchs, Y. Xu, J. Tan, L. Zhang, J. Xu, L. L. S. Keenan, J. Vitous, M. Ridges, E. M. Spinelll, M. Johnson, J. Org. Chem. 2006, 71, 7133-7145.

[24] V. Farina, C. Shu, X. Zeng, X. Wei, Z. Han, N. K. Yee, C. H. Senanayake, Org. Process Res. Dev. 2009, 13, 250-254.

[25] H. Wang, H. Matsuhashi, B. D. Doan, S. N. Goodman, X. Ouyang, W. M. Clark, Jr., Tetrahedron 2009, 65, 6291-6303.

[26] B. R. Galan, K. P. Kalbarczyk, S. Szczepankiewicz, J. B. Keister, S. T. Diver, Org. Lett. 2007, 9, 1203-1206.

[27] M. Lindmark-Hamberg, K. B. Wagener, Macromolecules 1987, 20, 2949-2951.

[28] S. E. Lehman, K. B. Wagener, in Handbook of Metathesis (Ed.: R. H. Grubbs), Wiley-VCH, Weinheim, 2003, Ch. 3.9, pp. $283-$ 353.

[29] T. W. Baughman, K. B. Wagener, in Advances in Polymer Science, Vol. 176 (Ed.: M. Buchmeiser), Springer, Berlin, 2005, pp. $1-42$.

[30] M. A. Hillmyer, R. H. Grubbs, Macromolecules 1993, 26, 872 874.

[31] M. K. Mahanthappa, F. S. Bates, M. Hillmyer, Macromolecules 2005, 38, 7890-7894.

[32] S. M. Banik, B. L. Monnot, R. L. Weber, M. K. Mahanthappa, Macromolecules 2011, 44, 7141-7148.

[33] X. Michel, S. Fouquay, G. Michaud, F. Simon, J.-M. Brusson, J.-F. Carpentier, S. M. Guillaume, Polym. Chem. 2016, 7, 4810-4823.

[34] X. Michel, S. Fouquay, G. Michaud, F. Simon, J.-M. Brusson, P. Roquefort, T. Aubry, J.-F. Carpentier, S. M. Guillaume, Polym. Chem. 2017, 8, 1177-1187.

[35] C. W. Bielawski, D. Benitez, T. Morita, R. H. Grubbs, Macromolecules 2001, 34, 8610-8618.

[36] M. R. Radlauer, M. E. Matta, M. A. Hillmyer, Polym. Chem. 2016, 7, 6269-6278.

[37] H. Katayama, H. Urushima, F. Ozawa, Chem. Lett. 1999, 28 , $369-370$

[38] H. Katayama, Y. Fukuse, Y. Nobuto, K. Akamatsu, Macromolecules 2003, 36, 7020-7026.

[39] P. Liu, M. Yasir, A. Ruggi, A. F. M. Kilbinger, Angew. Chem. Int. Ed. 2018, 57, 914-917; Angew. Chem. 2018, 130, 926-929.

[40] M. Yasir, P. Liu, I. K. Tennie, A. F. M. Kilbinger, Nat. Chem. 2019, 11, 488-494.

[41] R. A. Lyndon, G. Zwfifel, Synthesis 1974, 658-659.

[42] D. P. White, J. C. Anthony, A. O. Oyefeso, J. Org. Chem. 1999, 64, 7707-7716.

[43] J. B. Matson, S. C. Virgil, R. H. Grubbs, J. Am. Chem. Soc. 2009, $131,3355-3362$. 